\title{
Textos tecnológicos no ensino médio de física
}

\author{
Technological texts in high school physics teaching
}

José Cláudio Santiago ${ }^{1}$

\section{RESUMO}

Após as sucessivas reformas no sistema de Ensino Médio, muito se tem falado sobre a tecnologia como saber necessário para a formação básica. Situados no mesmo quadro, os livros didáticos de ciências esforçamse por inserir conteúdos relacionados à tecnologia em seus textos. Realizamos uma pesquisa bibliográfica e empírica para entendermos em que medida estes textos contribuiriam para a concretização de propostas educacionais relativas à tecnologia. Obtivemos resultados a partir da observação da relação de cada texto da amostra com algumas propostas educacionais para o ensino da Física por meio da tecnologia. Verificamos que há uma significativa quantidade de textos relacionados à tecnologia que já não podem mais ser considerados como textos complementares. Ou seja, houve algum avanço nos últimos anos. No entanto, há necessidade de reformulação e de ampliação da quantidade de textos efetivamente tecnológicos, por assim dizer.

Palavras-chave: Textos tecnológicos, livros didáticos, Diagrama de Fluxo.

\section{ABSTRACT}

After many reforms in the high-school teaching system, it has been talking about technology as necessary knowledge for basic education. Located in this context, science textbooks struggle to insert in these books knowledge related to technology. In this work, we came to results from the relationship of each collected text with some educational purposes for Physics teaching by means of the technology. As we have verified, there is significant amount of text related to technology that cannot be considered complementary texts. So, many books have been moving forward in the last few years. In spite of this advances, there is still a need for increasing and reform of the effective technological texts, as way of saying.

Keywords: Technological texts, Textbooks, Diagram of Flux.

1 Universidade Americana. 


\section{INTRODUÇÃO}

Após as últimas reformas no sistema de Ensino Médio (EM), muito se tem falado sobre a tecnologia como saber necessário para a formação básica. Situados no mesmo contexto, os livros didáticos (LDs) de ciências, mais a rigor, esforçam-se por inserir em seus textos conteúdos relacionados à tecnologia. Ainda que a denominação não seja usual, parece que os " textos tecnológicos ” não têm sido objeto de preocupação mais pronunciada por parte dos autores, dos professores e muitos dos agentes envolvidos no processo de seleção e avaliação dos LDs. Observações à parte, o fato é que os textos relacionados à tecnologia estão presentes nas coleções didáticas. Mas a questão seria se estes textos são mesmo tecnológicos. Passando da questão para a afirmação, o problema estaria em que precisamos despertar a atenção para a necessidade de uma identidade para estes textos. Afinal, não deveríamos aceitar que a simples inserção de automóveis, de instrumentos hospitalares ou outros produtos da atividade tecnológica, representados em LDs de ciências, possam ser confundidos com a representação do conhecimento tecnológico nestas coleções. Possivelmente, para compreendermos o texto tecnológico, antes, deveríamos compreender melhor a própria tecnologia. Pois a sua interpretação como campo de conhecimento, atividade (BUNGE, 1985) ou mesmo como um modo de vida (BORGMANN, 1984, apud CUPANI, 2004, pp. 497-499), poderiam abrir espaços para avaliação do que de fato vem ocorrendo nos LDs de ciências em relação à tecnologia. Para orientarmos a nossa preocupação para aspectos mais específicos, restringimos nossas dúvidas à seguinte questão: Da forma como se apresentam nos LDs de Física do EM, os textos tecnológicos contribuem para a concretização das propostas educacionais relativas à tecnologia? No curso deste trabalho, desenvolvemos uma pesquisa bibliográfica, empírica e originada a partir dos LDs de Física por corresponder à nossa atuação mais específica no ensino das ciências.

\section{REVISÃO DE LITERATURA}

A pesquisa a respeito dos conhecimentos e publicações relacionados à nossa questão esteve alicerçada na consulta ao Banco de Dados do Portal de Periódicos da Coordenação de Aperfeiçoamento de Pessoal de Nível Superior (CAPES/MEC). Os descritores empregados foram "livros didáticos”, "livros” e/ou "tecnologia”, considerando as variações dos termos. Ainda, observamos diretamente os seguintes periódicos: Revista Brasileira de Ensino de Física, Caderno Brasileiro de Física, Revista Brasileira de Ensino de Ciências e Tecnologia, Teses e Dissertações do Programa de Pós Graduação em Educação Científica e Tecnológica da Universidade Federal de Santa Catarina (UFSC), Revista Ciência\&Educação, Revista Electrónica de Enseñanza de las Ciencias, International Journal of Science and Mathematics Education, Programa de Pos-Graduação Interunidades em Ensino de Ciências - Universidade de São Paulo (USP). Pesquisamos as publicações situadas entre os anos de 1995 e 2012. Ou seja, desde o ano anterior à Lei de Diretrizes e Bases da Educação Nacional LDB/96.

\section{Da Filosofia e Ensino da Tecnologia aos textos tecnológicos}

Um momento referencial no desenvolvimento dos estudos da Filosofia da Tecnologia, de forma mais sistematizada, encontra-se na década de 90. É o período em que, Mitcham et. al. (1990) discutem e introduzem diversas questões referentes à metodologia de investigação tecnológica ressentindo-se da falta de estudos filosóficos necessários ao desenvolvimento desta área de pesquisa. No curso desse período, Guerrero et. al. (1990, p. 28) argumentam que "os epistemólogos direcionaram suas preocupações para o desenvolvimento de estudos em Filosofia da Ciência ao tempo em que acabaram por deixar de lado a ciência aplicada e a tecnologia”. Ele destaca a visão de Friedrich Rapp (1981) segundo a qual a falta de estudos sistemáticos sobre a técnica científica moderna seria advinda da reflexão filosófica tradicional que releva a concepção filosófica de homem como animal racional. E assim, o estudo da técnica moderna passaria a ser visto como um campo de estudos de menor relevância. Rapp (1981) assinala ainda que Bunge (1969) e a maioria dos epistemólogos identificam a tecnologia com ciência aplicada. Consideram, no entanto, essa identificação simplista e parcial por desconsiderar as componentes da tecnologia que se relacionam com as teorias da ciência pura e com as teorias da ciência aplicada. 
Para Cerezo e Luján (1998), a tecnologia é o produto do conhecimento tecnológico e dos fatores próprios da atividade humana. Estes autores atribuem aos conhecimentos tecnológicos, dimensões de conhecimento tácito e de conhecimento codificado. O primeiro refere-se às experiências e juízos pessoais do indivíduo imerso na atividade tecnológica, ao passo que, o segundo, diz respeito às atividades científica e técnica, podendo haver imbricações.

Para Cerezo e Luján (1998), a Filosofia da Tecnologia já se estabeleceu como um campo de estudos autônomo. Já dispõe, portanto, de suas revistas especializadas, autores destacados e suas próprias tradições. Ainda assim, vale ressaltar que, para esses autores, a Filosofia da Tecnologia, como campo de estudos, estabeleceu-se enquanto subdisciplina filosófica, tendo ampliado o seu espaço de atuação ao se vincular aos estudos CiênciaTecnologia-Sociedade (CTS). Por sua vez, os estudos CTS têm se constituído atualmente numa das abordagens centrais que justificam o estabelecimento da educação tecnológica.

Gilbert (1995), a partir de Medway (1989) e Layton (1994), apresenta três argumentos que dão suporte para a educação tecnológica. Estes seriam os econômicos, os sociais e os educativos. Os argumentos econômicos seriam todos aqueles fundamentados na importância da tecnologia para o desenvolvimento de atividades que vão trazer riquezas para todos os países. Tais argumentos estariam orientados para a visão de que a educação tecnológica poderia proporcionar aos jovens a preparação para o mundo do trabalho. Em oposição ao referido argumento estaria o de que a colocação no mercado de trabalho industrial é de natureza provisória e portanto deveria se enfatizar as habilidades básicas como leitura, escrita e aritmética bem como conhecimentos que permitiriam interação social mais ampla tal como o estudo da geografia e da história nacional. Os argumentos sociais dizem respeito à preocupação com a consciência que deveria existir a respeito do desenvolvimento tecnológico e o uso de capacidades relacionadas ao direcionamento e controle de impactos sócio-ambientais. Os argumentos educativos destacam o fato de que a importância da educação tecnológica estaria em proporcionar aos jovens o contato com a tecnologia por esta constituir-se num dos produtos da ação humana.

Ao nos referirmos aos LDs de ciências do Ensino Médio, podemos buscar compreender também algumas teorias de aprendizagem relevantes para o ensino tecnológico. Ou seja, localizar nas coleções didáticas elementos destas teorias que reflitam, de certa forma, a intenção do autor no que diz respeito aos seus objetivos de aprendizagem. Ausubel (1968) apresenta a teoria da aprendizagem significativa tomando por base a interação entre o material a ser apreendido e o conhecimento que o aluno já possui. Nesse sentido, a aprendizagem significativa relaciona-se claramente com o que atualmente denominamos conhecimentos prévios e conhecimentos do cotidiano.

A observação atenta da literatura que trata da Filosofia da Tecnologia permite perceber que o conhecimento científico, a perícia técnica (know-how) e o valor social são referidos por muitos autores que discutem aspectos inerentes ao conhecimento tecnológico. Na presente discussão, a linguagem associada ao conhecimento tecnológico se refere a atributos tais como planejamento, projetos, eficiência, pesquisa, desenvolvimento entre outros. A partir da aplicação dessas noções, podemos adotar o entendimento de que um texto tecnológico contém em sua estrutura o conhecimento científico, o conhecimento da perícia técnica e ressalta a importância social associada ao seu conteúdo. Além do mais, se refere a aspectos econômicos, materiais, de planejamento, técnicos e sociais, entre outros, entendidos, em seu conjunto, como múltiplos aspectos da atividade tecnológica. Uma possível categorização para os referidos aspectos tecnológicos poderia classificá-los em : a) dimensão material; b) dimensão econômica; c) dimensão geradora; d) dimensão preparativa; e) técnico-científica; e) de pesquisa; f) de importância social. Portanto, acreditamos que um texto razoável em tecnologia no contexto educativo seria, no mínimo, bem contextualizado e com um nível de informações que estão além da simples ilustração do conteúdo anteriormente apresentado. Assim, não deve ser visto como um texto auxiliar e nem como complementar ao conteúdo científico. Antes, pode ser um texto principal estabelecido por uma identidade própria, de forma a evidenciar melhor sua própria importância. É então nesse sentido que nos referimos aos textos tecnológicos no decorrer de todo presente trabalho. 


\section{FUNDAMENTOS METODOLÓGICOS}

Os fundamentos metodológicos tomaram por base o uso de um diagrama de fluxo (adaptado). Tal instrumento foi empregado por Santiago, Guimarães e Costa (2012) para relacionar propostas de atividades experimentais no ensino de ciências com as propostas presentes nos documentos oficiais do Ensino Médio, conforme figura 1. Como o uso do diagrama de fluxo diz respeito aos vínculos entre informações, realizamos uma adaptação de forma a empregá-lo na presente pesquisa. Aqui, foram estudadas as interações entre os textos da amostra e os critérios associados às propostas, conforme o quadro 1 , mais adiante.

O diagrama de fluxo (adaptado) toma por base a ideia de que os critérios (quadro 1) poderiam servir de meio para interligar os textos tecnológicos da amostra às propostas apresentadas nos Parâmetros Curriculares Nacionais para o Ensino Médio (BRASIL, 2000, p. 96). Assim, por exemplo, se um determinado texto analisado está em acordo com um determinado critério, esta relação é então representada por um segmento de reta unindo o número atribuído ao texto ao número atribuído ao critério. De acordo com a linguagem que estamos empregando, cada ligação representa uma unidade de fluxo. Entre as diversas competências encontradas nos PCNEM (2000, p. 96), foram escolhidas sete entre elas. Ou seja, foram escolhidas aquelas que mais diretamente dizem respeito ao conhecimento tecnológico. Assim, cada canal (aqui referidos como critério) pode ser entendido como um elo que permite vincular um ou mais textos a uma determinada proposta ou objetivo.

\section{PROPOSTAS EDUCACIONAIS}

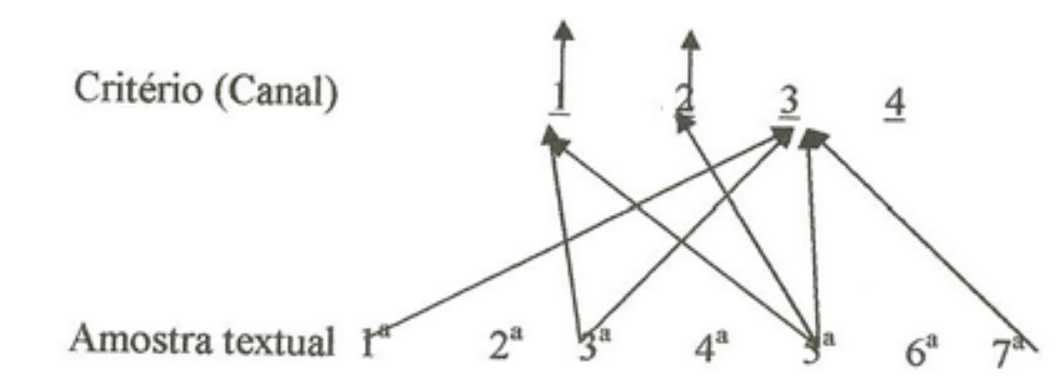

Figura 1 - Diagrama de Fluxo (adaptado) - Fonte: Santiago, Guimarães e Costa, 2012, pp.75-93.

Portanto, na figura 1, representamos geometricamente o fluxo proporcionado por um subconjunto de textos prováveis em atingir uma determinada proposta educacional através de um conjunto de canais. Considerando-se um conjunto de textos analisados, o número de textos prováveis Np corresponde à quantidade de textos relacionados ao conjunto de critérios. Este conjunto de critérios permite o fluxo de cada texto Np para determinada proposta. Por exemplo, no diagrama acima, haveria apenas quatro textos prováveis: o primeiro, o terceiro, o quinto e o sétimo. Isto é $\mathrm{Np}=4$. E, teríamos o total de sete textos $(\mathrm{T}=7)$ sobre um determinado conteúdo, que em nosso trabalho corresponde à tecnologia.

Podem-se associar dois tipos de probabilidade ao diagrama de fluxo: a probabilidade de contribuição mínima e a probabilidade de contribuição efetiva para a concretização das propostas.

\section{Probabilidade de Contribuição Mínima, $P_{\min }$}

Neste caso, quer-se saber a partir de um número total de textos analisados T, qual a quantidade que pode chegar, no mínimo, a um entre os critérios. E então podemos considerar

$$
\text { Pmín = Np / T. }
$$


Para calcular o valor numérico de Pmín, não é relevante considerar se o fluxo produzido por um determinado texto está associado a um ou a diversos critérios. Se fosse construído no decorrer da pesquisa um diagrama de fluxo tal como o apresentado na fig. 1, a probabilidade de contribuição mínima dos textos tecnológicos para a concretização das propostas educacionais seria de 4/7, ou Pmín = 57,1 \%. Devemos observar ainda que a probabilidade mínima não se refere necessariamente à menor probabilidade. O termo mínimo é empregado no sentido de que, para ser provável, o texto deve atingir, no mínimo, um dos critérios.

\section{Probabilidade de Contribuição Efetiva, Pef.}

O último exemplo acima deixa claro que no cálculo da probabilidade mínima, todos os textos prováveis contribuem igualmente para um determinado objetivo, já que no cálculo de Pmín o fluxo divergente total proporcionado pelo texto não é relevante. Entretanto, observa-se, por exemplo, que enquanto o primeiro texto provável (no diagrama) produz um fluxo unitário, o quinto produz um fluxo de 3 unidades. Essa diferença faz ver que a definição anterior não leva em consideração a contribuição individual de cada texto. Assim, muitas vezes um texto contribui mais que outro para se concretizar os objetivos. Essas informações nos levam a buscar o auxílio de mais outra equação que considere esta diferença. Para satisfazer essa situação, podemos empregar a equação

$$
\mathrm{Pef}=\mathrm{S} / \mathrm{Np} \cdot \mathrm{Nc} \quad(\mathrm{Nc}>1)
$$

Nessa equação, S representa o fluxo total e Nc representa o número de critérios.

Para o diagrama de fluxo apresentado, como exemplo na fig. 1, existem quatro critérios ( $\mathrm{Nc}=4$ ), sendo que o fluxo total seria de sete unidades $(S=7)$. Assim, a probabilidade de contribuição efetiva do conjunto dos textos para a concretização de determinada proposta seria $\mathrm{Pef}=43,7$ \%.

\section{O EMPREGO DA METODOLOGIA}

No quadro 1, apresentamos as propostas de competências e habilidades básicas para os alunos do EM no que diz respeito a tecnologia, de acordo com o PCNEM (2000, p. 96). Na coluna ao lado, apresentamos uma possível formulação de critérios que permitem relacionar o texto à correspondente proposta. Apenas a resposta positiva para a questão apresentada permitiu a construção da unidade de fluxo. Isto é, a ligação do texto ao critério por meio de uma seta, conforme o diagrama de fluxo.

Quadro 1 - Critérios propostos para relacionar cada texto à correspondente proposta.

\begin{tabular}{|l|l|}
\hline PROPOSTA & CRITÉRIOS \\
\hline $\mathbf{1}$ Entender as relações entre o desenvolvimento das & $\mathbf{1}$ O texto tecnológico possui conteúdos das \\
Ciências Naturais e o desenvolvimento tecnológico. & disciplinas Física, Química e Biologia? \\
$\mathbf{2}$ Associar as diferentes tecnologias aos problemas & $\mathbf{2}$ O texto está relacionado à situações ou \\
que se propuseram e se propõem solucionar. & problemas relevantes ainda não resolvidas? \\
$\mathbf{3}$ Entender o impacto das tecnologias associadas às & $\mathbf{3}$ O texto apresenta-se contextualizado \\
Ciências Naturais na sua vida pessoal. & com situações do cotidiano? \\
$\mathbf{4}$ Entender o impacto das tecnologias associadas & $\mathbf{4}$ O texto refere-se a processos de produção? \\
às Ciências Naturais nos processos de produção. & $\mathbf{5}$ O texto apresenta quantidade significativa de \\
$\mathbf{5}$ Entender o impacto das tecnologias associadas & informações relevantes para a construção do \\
às Ciências Naturais no desenvolvimento & conhecimento? \\
do conhecimento. & $\mathbf{6}$ O texto refere-se explicitamente \\
$\mathbf{6}$ Entender o impacto das tecnologias associadas & ao contexto da vida social? \\
ás Ciências Naturais na vida social. & $\mathbf{7}$ O texto permite compreender como a matemática \\
$\mathbf{7}$ Compreender conceitos, procedimentos e & pode ser aplicada ao contexto da tecnologia? \\
estratégias matemáticas, e aplica-las a situações & \\
diversas do contexto das tecnologias. & \\
\hline
\end{tabular}


Consideramos relevante para a pesquisa que os livros didáticos fossem obras constituintes do Programa Nacional do Livro Didático para o Ensino Médio (PNLEM-2009) e do Programa Nacional do Livro Didático (PNLD-2012). Ainda, os livros didáticos foram escolhidos considerando também que já tínhamos certa familiaridade em seu manuseio. As obras empregadas na pesquisa foram:

GASPAR, A. Física. Volume único. São Paulo, SP: Editora Ática, 2009.; GASPAR, A. Compreendendo a Física: Volume único. São Paulo, SP: Editora Ática, 2012.; LUZ, A. M. R.; ALVARENGA, B. A. Física: ensino médio. São Paulo, SP: Editora Scipione, 2009. 3 v.; LUZ, A. M. R.; ALVARENGA, B. A. Curso de Física. São Paulo, SP: Editora Scipione, 2012. 3 v.; SAMPAIO, J. L. P.; CALÇADA, C. S. V. Universo da Física. 2 ed. São Paulo, SP: Editora Saraiva, 2009. 3 v.; SAMPAIO, J. L. P.; CALÇADA, C. S. V. Universo da Física: volume único. 2 ed. São Paulo, SP: Editora Saraiva, 2009.; SANT`ANNA, B.; MARTINI, G.; REIS, H.C.; SPINELLI, W. Conexões com a Física. São Paulo, SP: Editora Moderna, 2010. 3 v.; TORRES, C. M. A.; PENTEADO, P. C. M. Física: Ciência e Tecnologia. São Paulo, SP: Editora Moderna, 2005. 3 v.; TORRES, C. M. A.; PENTEADO, P. C. M. Física: Ciência e Tecnologia. 2 ed. São Paulo, SP: Editora Moderna, 2012. 3 v.

Para compor a amostra, foram selecionados dez textos tecnológicos considerados como aqueles que abordam a tecnologia em seus múltiplos aspectos. Os textos selecionados foram:

Texto 34; O que diz a mídia: carros de alumínio; Física, Ciência e Tecnologia, vol.1, p.112, 2012; Física. Texto 42; O motor de combustão; Física, Ciência e Tecnologia, vol.2, p.113, 2012; Física. Texto 49; A energia solar e a captação por meio de coletores; Física, Ciência e Tecnologia, vol.3, pp. 186-189, 2012; Física. Texto 50; A energia da biomassa; Física, Ciência e Tecnologia, vol.3, pp. 197-199, 2012; Física. Texto 58; Os computadores e a internet; Física, Ciência e Tecnologia, vol.3, pp. 325-330, 2012; Física. Texto 60; Para saber mais: Sem atrito?; Conexões com a Física, vol.1, p.202, 2012. Física. Texto 93; A nanotecnologia; Conexões com a Física, vol.3, pp. 384-386, 2012. Física. Texto 100; Tópico Especial; A fibra ótica; Curso de Física, vol. 2, p. 214, 2012; Física. Texto 102; Tópico Especial; Transistor; Curso de Física, vol. 3, pp. 176-181, 2012; Física. Texto 110; Conhecendo um pouco mais; História dos termômetros e das escalas termométricas; Física Alberto Gaspar, vol. 2, p. 301-308, 2012; Física.

\section{RESULTADOS E DISCUSSÕES}

Adotamos sete critérios $\left(\mathrm{Nc}_{\mathrm{c}}=7\right)$ como canais, conforme o quadro 1 . O conjunto formado por todos os textos da amostra produziu um fluxo total de 43 unidades (tabela 1), isto é, $\mathrm{S}=43$. A partir destas variáveis, obtivemos as probabilidades mínima e efetiva. Isto então resultou em Pmín $=100 \%$ e em Pef $=62.9 \%$. Para o fluxo por meio de cada um dos critérios foram obtidos respectivamente N1 = 2, N2 = 6, N3 = 5, N4 = 6, N5 = 10, N6 $=9$ e N7 $=5$.

Tabela 1 - Resultados obtidos a partir do diagrama de fluxo.

\begin{tabular}{|l|l|l|l|l|l|}
\hline Np & \multicolumn{2}{|l|}{ Pmín (\%) } & \multicolumn{2}{l|}{ Pef (\%) } & Total (S) \\
\hline & & & & & \\
\hline 10 & 100 & & 62.9 & & 43 \\
\hline
\end{tabular}

Fonte: Organizado pelo autor.

Para a probabilidade mínima encontrada, $100 \%$, o resultado apenas significa que todos os textos escolhidos para a amostra foram textos prováveis. O resultado reflete a própria natureza da amostra já que, para esta, havíamos escolhido os textos que abordam o maior número possível de aspectos da tecnologia.

O resultado correspondente ao sétimo critério não se mostra tão pronunciado. Entendemos que esse critério se refere de maneira mais direta à evidência de aprendizagem por se referir à capacidade de aplicar o conhecimento adquirido a situações novas, em acordo com a teoria da aprendizagem significativa (AUSUBEL, 1968). 
Ou seja, encontramos nos textos tecnológicos de Física do EM pouco incentivo para o desenvolvimento da capacidade de compreender como a Matemática pode ser aplicada a situações diversas do contexto das tecnologias. De acordo com o resultado N1 = 2, há evidências de baixo nível de interdisciplinaridade, ao mesmo tempo em que quase todos os textos se referem à vida social. Este conjunto de resultados impele a observarmos que a abordagem apresentada nos textos não está muito distante de associar a tecnologia a um modo de vida moderno em cujo centro estariam os produtos e seu consumo. Ou seja, os textos da amostra apresentam um enfoque para a tecnologia muito mais em acordo com a visão de Borgmann (1984), distanciando-se da visão de Bunge (1985) e Mitcham (1990). Isto é, os enfoques relacionados a uma visão estruturante da tecnologia encontram-se distantes dos textos pesquisados. Essa visão seria necessária para evidenciarmos o nível incentivo à aprendizagem sobre as tecnologias.

A probabilidade efetiva encontrada, 63 \%, expressou assim o nível de comunicação dos textos tecnológicos com referidas propostas. Assim, os textos que abordam a tecnologia em seus múltiplos aspectos contribuem num nível significativo para a concretização das propostas educacionais relativas à tecnologia. Ou seja, os textos tecnológicos apresentam em sua estrutura um número significativo de informações diretamente vinculadas às habilidades e competências relativas à tecnologia propostas pelos PCNEM (2000, p. 96). Cabe assinalar que cinco (do $1^{\circ}$ ao $5^{\circ}$ ) entre os dez textos da amostra trazem em sua estrutura todas as categorias da atividade tecnológica. Isto é, 7 categorias. Os outros cinco (do $6^{\circ}$ ao $10^{\circ}$ ) transportam 6 categorias da atividade. Devemos observar ainda que o resultado percentual encontrado para a probabilidade efetiva, $\mathrm{P}_{\text {ef }}$, expressa portanto o nível máximo de vínculo com as propostas uma vez que a grande maioria dos textos transportam muito poucas categorias, caracterizando-se pelo empobrecimento da representação do conhecimento tecnológico em suas estruturas. Como se pode notar, entre os critérios observados, o primeiro apresentou a maior deficiência, caracterizando, portanto, a necessidade de inserção de abordagens que reforcem a interdisciplinaridade dos textos. O quinto critério foi o que apareceu de forma mais pronunciada. Assim, o resultado $\mathrm{N}_{2}=10$ mostra que todos os textos da amostra, estão diretamente relacionados ao quinto critério. Ou seja, os textos tecnológicos apresentam quantidades significativas de informações relevantes para a construção do conhecimento. Esta conclusão parece decorrer naturalmente da própria reformulação dos processos de seleção e aprovação dos LDs para atender às orientações apresentadas pelas propostas curriculares oficiais em relação a inserção da tecnologia nas coleções didáticas.

\section{CONSIDERAÇÕES FINAIS}

No decorrer da pesquisa, observamos que ainda são muito poucos os textos que abordam a tecnologia em seus múltiplos aspectos. E que, a grande maioria dos textos relacionados à tecnologia ainda caracterizam-se por apresentar uma visão restrita do conhecimento tecnológico ao confundi-los com os produtos da atividade tecnológica. Verificamos que já há uma significativa quantidade de textos relacionados à tecnologia que já não podem mais ser considerados como textos complementares ao conteúdo principal. Entendemos, portanto, que houve avanço nos últimos anos ao se reforçar nos LDs de Física a presença do conhecimento tecnológico. Porém, este avanço ainda está longe de ser suficiente para considerarmos que o conhecimento tecnológico esteja sendo trabalhado no Ensino Médio por meio das obras didáticas. Ainda, para que as coleções didáticas transportem, entre suas páginas, textos efetivamente tecnológicos, há necessidade de reformulação de diversos textos atuais. E, para melhor incentivo ao aprendizado da tecnologia por meio dos livros didáticos, sinalizamos a necessidade de ampliação da atual quantidade de textos mais efetivamente tecnológicos, por assim dizer. 


\section{REFERÊNCIAS}

AUSUBEL, D.P. Educational Psychology: A Cognitive View. New York: Holt, Rinehart and Winston, 1968.

BORGMANN, A. Technology and the character of contemporary life. A philosophical inquiry. Chicago: University of Chicago Press, 1984. 310 p.

BRASIL. Lei de Diretrizes e Bases da Educação Nacional: Lei 9.394, de 20/12/1996.

Ministério da Educação, Secretaria da Educação Média e Tecnológica. Parâmetros Curriculares Nacionais: ensino médio. Brasília: Ministério da Educação, p. 4, 2000.

Secretaria da Educação Média e Tecnológica. PCN+ Ensino Médio: orientações educacionais complementares aos Parâmetros Curriculares Nacionais. Ciências da Natureza, Matemática e suas Tecnologias. Brasília: MEC, SEMTEC, 2002.

BUNGE, M. La investigación científica. Barcelona: Ariel, 1969.

BUNGE, M. Treatise on Basic Philosophy. Dordrecht: Dordrecht Reidel Publishing Company, 1985. v. 7: Epistemology and Methodology.

CEREZO, J. A. L.; LUJÁN, J. L. Filosofía de la Tecnología. Revista internacional de filosofia, v. 27, n. 3, 1998. Disponível em http://www.oei.es/salactsi/teorema00.htm （Acesso em Jan. 2012).

CUPANI, A. A tecnologia como problema filosófico: três enfoques. Scientiae Studia, São Paulo, v. 2, n. 4, p. 493-518, 2004.

GILBERT, J. K. Educación Tecnológica: uma nueva asignatura em todo el mundo. Enseñanza de las ciências: revista de investigación y experiências didacticas, v. 13, n. 1, p. 15-24, 1995.

GÓMEZ, M. M. Filosofía versus técnica. In: MITCHAM, C.;BORRERO, M. M. P. (Eds). El nuevo mundo de la filosofia y la tecnología. University Park, PA: STS PRESS, 1990. p. 34-42.

GUERRERO, G. B. La metódica de la investigación tecnológica. In: MITCHAM, C.; BORRERO, M. M. P. (Eds). El nuevo mundo de la filosofia y la tecnología. University Park, PA: STS PRESS, 1990. pp. 28-33.

LAYTON JÚNIOR, E. T. Technology as knowledge. Technology and Culture, v. 15, n. 1, p. 31 - 41, Jan. 1994.

MEDWAY, P. Issues in the theory and practice of technology education. Studies in Science Education, 16, pp. 1 $-24,1989$.

MITCHAM, C. et. al. El nuevo mundo de la filosofia y la tecnología. University Park, PA: STS PRESS, 1990.

RAPP, F. Filosofía analítica de la técnica. Buenos Aires. Editora Alfa, 1981. 211 p.

SANTIAGO, J. C. R. GUIMARÃES, E. M.; COSTA, I. F. da. A experimentação nos livros didáticos de Física do Ensino Médio e os objetivos educacionais. In: GUIMARÃES, E. M.; CAIXETA, J. E. Trilhas e encontros: mediações e reflexões sobre o ensino de ciências. Curitiba: Editora CRV, 2012. p. 75-93. 